\title{
Effect of inorganic solids, wax to asphaltene ratio, and water cut On the Stability of Water-in-Crude Oil Emulsions
}

\author{
Adel M. Elsharkawy ${ }^{* 1}$, Tahar A. Al-sahhaf ${ }^{2}$, Mohamed A. Fahim ${ }^{2}$, \\ ${ }^{1}$ Department of Petroleum engineering, ${ }^{2}$ Department of Chemical Engineering \\ College of Engineering and Petroleum, Kuwait University \\ P.O. Box 5969, Safat 13060, Kuwait
}

\begin{abstract}
The formation of stable water-in-crude oil emulsions during petroleum production and refinery may create sever and costly separation problems. It is very important to understand the mechanism and factors contributing to the formation and stabilization of such emulsions for both great economic and environmental development.

This paper investigates some of the factors controlling the stability of water-in-crude oil emulsions formed in Burgan oil field in Kuwait. Water-in-crude oil emulsion samples collected from Burgan oil filed have been used to separate asphaltenes, resins, waxes, and crude oil fractions. These fractions were used to prepare emulsion samples to study the effect of solid particles $\left(\mathrm{F}_{3} \mathrm{eO}_{4}\right)$ on the stability of emulsions samples. Results indicate that high solid content lead to higher degree of emulsion stability. Stability of emulsion samples under various waxes to asphaltenes (W/A) ratios have also been tested. These tests showed that at low W/A content, the emulsions were very stable. While at a wax to asphaltene ratio above 1 to 1 , the addition of wax reduced emulsion stability. Stability of emulsion samples with varying amount of water cut has also been investigated. Results indicated that stability and hence viscosity of emulsion increases as a function of increasing the water cut until it reaches the inversion point where a sharp decline in viscosity takes place. This inversion point was found to be approximately at $50 \%$ water cut for the crude oils considered in this study.
\end{abstract}

Key words: Asphaltenes; Resins; Waxes; Water-Oil Emulsions; Kuwaiti oil fields

\section{Introduction}

During production of conventional as well as heavy oils, emulsions occur when crude oil mix with produced formation water or injected water in reservoir, at well bore, in pipelines, and at surface facilities. These emulsions are undesirable because they cause high pressure drop due to their high viscosity. They also cause serious corrosion problems due to presence of chlorides dissolved in water (Yan et al., 2001).

Crude oils are very complex fluids comprising colloidal particles such as asphaltene and resins aggregates, dispersed in solution of mixed aliphatic/aromatic nature. Asphaltenes are the heaviest and most polar component of crude oils, are present in the form of colliodally dispersed particles. It is insoluble in $\mathrm{n}$ heptane and soluble in toluene. Asphaltenes have a molecular weight ranges from 800 to 4000 or even higher (Ramos et al., 2001; Spiecker et al., 2003; Elsharkawy et al., 2005). The asphaltenes are flat sheets of condensed polyaromatcic hydrocarbons linked together by sulfide, ether, and aliphatic chain groups. The edges of the sheets are alkyl chains. The polar parts of the asphaltene molecules interact with each others forming aggregates or micelles. As such, these micelles are very much polar. Resins are less polar and made up of smaller molecules with one end being hydrophilic made up of functional groups and the other end hydrophobic made up of alkyl chains. In crude oils, the resins are attached to the asphaltene micelles at the polar end and the non-polar end of the resin interact with crude oil. The resins solvate the asphaltene aggregates and keep them in colloidal suspension in the oil. The aromaticity of the oil also increases the solvency of the asphaltene aggregates. The colloidal aggregates can be interfacially active if the interactions of the asphaltenes with the water interface are favored over interaction with resins and aromatics in the crude oil phase. It is proposed that asphaltenes stabilize water-in-oil emulsion if they are near or above the asphaltene precipitation onset point (Gafonova and Yarranton, 2001). This is the point at which the asphaltene start to precipitate, caused by addition of an alkane such as n-heptane to the crude oil.

It has been found experimentally that strong films made mainly of asphaltene with some resins were responsible for stabilizing water-in-oil emulsions. Resins although they are surface active, they can not stabilize emulsion alone. However, resins can adsorb quickly to water droplet interface, slowing coalescence and providing enough time for asphaltene to migrate to the interface and adsorb on the surface

* Corresponding author, Professor Adel Elsharkawy

E-mail address: asharkawy@kuc01.kuniv.edu.kw

Tel.: +965-483-6059; fax: +965-484-9558. 
forming the rigid film which prevents coalescence. Therefore, any factor which result in the disturbance of the asphaltene-asphaltene interaction will make the interfacial film weaker and make the droplet coming together easier.

Several studies have shown that the most important factor for emulsion stability relates to the asphaltene content (Xia, et al., 2004; Yarranton et al., 2000; Aske et al., 2002; Mc Lean and Kilpatrick, 1997). Water-in crude oil emulsion can be formed even in the absence of asphaltene. Li et al (2004) reported the formation of stable water-in-paraffinic crude oil during alkaline-surfactant-polymer flooding using sodium hydroxide as alkali component to enhance oil recovery at on shore oil field at Daqing in China. It was found that sodium hydroxide reacts with fatty acids in the aliphatic fraction of crude oil and/or with fatty acids formed from slow oxidation of long chain hydrocarbons, and form soap like interfacially active components. These accumulate at the crude oil-water interface and contribute to the stability of water/oil emulsion.

Spiecker et al., (2003) studied the effect of increasing resins to asphaltene (separated from off-shore California, Safaniya, and Argentina) of model oil of heptane-toluene mixtures on the stability of water-inoil emulsion. They found that the addition of resins to asphaltenes reduced the aggregate size by disrupting the $\pi-\pi$ and polar bonding interactions between asphaltene monomers. Interaction of resins with asphaltenic aggregates rendered the aggregates less interfacially active and thus reduced emulsion stability. The smallest aggregate sizes observed and the weakest emulsion stability at high resins to asphaltene (R/A) ratios presumably corresponded to asphaltenic monomers or small oligomers strongly interacting with resins molecules.

Li et al. (2002) studied the effect of molecular size, aromaticity, aromatic condensation, and carbonyl group concentrations on the stability of water-in-oil emulsions. They reported that higher molecular weight, higher aromaticity, higher aromatic condensation and higher carbonyl group concentration per unit aromatic structure give higher emulsion stability. The presence of inorganic particles in the emulsion results in increasing emulsion stabilization (Yan et al., 1999; Ali and Alqam, 2000; Gu et al., 2002). Asphaltene and resins are adsorbed on the solid particles making them surface active. The role of solid in emulsion stabilization depends on the resins to asphaltene (R/A) ratio (Sullivan and Kilpatrick, 2002). At low R/A ratio, large asphaltene aggregates form thick interfacial film, bridging particles and preventing adsorption to water-oil interfaces because of bulky shape of the solid-asphaltene aggregates. At high R/A ratio, smaller asphaltene aggregates adsorb to the solid particles and migrate to the water-oil interface enhancing the stability of the emulsion. As the R/A increases further, the adsorption of asphaltene on the solid particles decreases. The amount of surface active aggregates decreases affecting emulsion stability (Mclean and Kilpatrick, 1997; Midttun et al., 2000; Salou et al., 1998).

Midttun et al. (2000) reported that depending on the type of the resins, adding of resins to asphaltene-stabilized w/o emulsion may reduce emulsion stability, increase emulsion stability, or have no significant effect of emulsion stability. They reported that high polarity resins tend to reduce emulsion stability. Schorling et al. (1999), however, found that the influence of resins in the presence of asphaltenes on the properties of emulsions is independent of the origin of resins.

The degree to which solids increases emulsion stability depends on several factors such as particle size, shape and morphology, density, concentration and surface coverage, and wettability (Gelot, et al., 1984; Yan et al., 1999, 2001; Tambe and Sharma,1993, 1994; Aveyard, et al., 2003). Sztukowski and Yarranton (2005) investigated the role of solids in the stability of oil filed emulsions. They found that emulsion stabilized by fine solids and asphaltenes were most stable at a 2:1 fraction area ratio of asphaltene to solids. There is a strong correlation between asphaltene content and emulsion tightness (Kokal, 2002). The presence of wax in addition to asphaltene and resins are known to promote the stabilization of waterin-oil emulsion (Yarranton et al., 2000; Khristove et al., 2000).

Yan et al. 2001 studied the stability of water-in-oil emulsion using fine solids such as Kaolinite clay particles treated with asphaltenes, hydrophilic and hydrophobic colloid silica, hydrophobic polystyrene latex micropheres as well as fumed silica dry powders treated with silanization. Their experimental results showed that hydrophilic colloidal silica could only stabilize oil-in-water emulsion for short time. If hydrophobic particles were suspended in the aqueous phase prior to emulsification, they could only produce oil-in-water emulsions. Only hydrophobic particles suspended in organic phase prior to emulsification could stabilize water-in-oil emulsion. The presence of solids also change emulsion viscosity. Volume fraction of dispersed phase, the amount of solids present, and the wettability of solids affects emulsion viscosity (Yaghi, 2003). 
Stability of emulsions is assessed in several ways. Fingas and Fieldhouse, (2004) classified waterin-crude oil into four states or classes: stable, mesostable, unstable, and entrained water. Of these, only the stable and mesostable are defined as emulsions. These are established by their stability over time, their appearance and by rheological properties. Stable emulsions remain stable for at least four weeks under laboratory and tank conditions. Mesostable emulsions remains as such less than 3 days under laboratory conditions. Unstable water-in-oil is characterized by the fact that the oil does not hold significant amount of water, and when it does, it is only for a short time. Entrained water-in-oil states remains as such for less tan 1 day under laboratory conditions. Entrained water-in-oil states are most often produced from heavy oils. Gafonova and Yarranton (2001) and Sullivan and Kilpatrick (2002) assessed emulsion stability by measuring the amount of water resolved from emulsion as a function of time via heating or centrifuge. Kokal and Wingrove (2000) measure the stability of emulsion by adding a specific concentration of demulsifier which will destabilize the emulsion and measure the amount of water separated from emulsion as a function of time. Another approach to measure the stability of emulsion is the determination of the critical electric field (CEF) for emulsion breakdown (Midttun et al., 2000; Sullivan and Kilpatrick, 2002; Aske et al., 2002).

Although of numerous research about factors controlling stability of oil field emulsions, there is a lack of work on inorganic and organic solids stabilized water-in-oil emulsion despite their importance. In this paper, we shall present experimental results related to water-in-oil emulsions stabilized by inorganic solids and organic solids. Various parameters, especially the effect the effect of increasing concentration of solid particles of $\mathrm{F}_{3} \mathrm{eO}_{4}$, changing wax to asphaltene ratio, and various water cuts are investigated.

\section{Experimental}

\subsection{Materials}

Crude oil samples from Burgan oil field- the second largest oil field of the world, BG 321, BG366, BG75 and RA4 are used in preparation of various emulsion samples considered in this study. Molar mass, density and elemental analysis of asphaltenes, resins, and crude oils used in preparation of emulsion samples are given in Table 1. Details of the procedures used to separate various fractions and measurements of their physical and chemical properties are described elsewhere (Elsharkawy et al, 2005). Organic solvents used are: toluene (99.5\% purity; Scharlau), n-heptane (99.9\% purity; Baker analyzed), benzene (100\% purity; baker analyzed), and methanol (99.8\% purity; Univar). All chemical were used without further purification. Distilled water provided by Kuwait University facility having a pH 6 was used in emulsion preparation for all samples. Silica gel 60 provided by Fluka was used to absorb the resin fraction.

\subsection{Equipments}

Shaking bath model SB-16 provided by Techne was used to homogenize crude oil samples before oil is used in preparation of the emulsion samples. Rotary evaporator model BUCHI R-124 was used to remove extraction solvents. PH meter model HNNA HI9017 was used to adjust the $\mathrm{pH}$ of the aqueous phase. Silverson laboratory mixer emulsifier model SL2 was used to prepare all emulsion samples and centrifuge model Labofuge provided by Seraeus Sepatech was used to separate water from emulsion samples.

\subsection{General procedure}

The oil phase (either crude oil or model oil) was mixed water using Silverson Lab mixer emulsifier for 1 minute at $9000 \mathrm{rpm}$. Equal volume of distilled water of $\mathrm{pH} 6$ was added slowly to oil samples for 5 minutes during mixing. Emulsion samples were transferred immediately to $10 \mathrm{ml}$ centrifuge tubes and left for $1.5 \mathrm{hr}$ at room temperature to settle down. The stability of settled emulsion was studied by measuring the amount of water separated from emulsion as a function of time for 12 hours. Centrifuge tubes were capped to prevent solvent evaporation and centrifuged at $4000 \mathrm{rpm}$ for 5 minutes then transferred to $60{ }^{\circ} \mathrm{C}$ water bath for 2 hours, centrifuge again for 5 minutes and heated for 2 hours. Successive heating and centrifuge was repeated for 12 hours or until no appreciable change in the amount of separated water is observed. The amount of water separated from emulsion was reported as the volume percent of initial water content of the emulsion sample. 


\subsection{Preparation of water-oil emulsions}

Various emulsion samples were prepared to study the effect of inorganic solid, wax to asphaltene ratio, and water cut to the stability of emulsion samples.

\subsubsection{Effect of inorganic solid}

Emulsions of different w/w percents $(0,0.2,0.4,0.6,0.8$, and $1 \%)$ of $\mathrm{Fe}_{3} \mathrm{O}_{4}$ in crude oils were prepared by mixing $(0,0.1,0.2,0.3,0.4$ and $0.5 \mathrm{~g})$ of $\mathrm{Fe}_{3} \mathrm{O}_{4}$ suspended in a specific volume of deionized water with equal volume of crude oil to study the effect on inorganic solid on the stability of water-in-oil emulsions.

\subsubsection{Effect of, wax to asphaltene ratio}

To study effect of changing wax to asphaltene ratio, emulsion samples of different weight ratios of wax to asphaltene were prepared by dissolving various amount of wax in crude oil. The wax was separated according to the procedure explained elsewhere (Elsharkawy et al, 2000). Various wax to asphaltene ratios from 0.5:1 to $4: 1$ have been used to prepare different emulsions using crude oil RA4 and crude oil BG75.

\subsubsection{Effect of water cut}

The effect of water cut on stability of emulsion samples has been investigated. Emulsions of different water cuts $(0,20,40,45,50$ and $60 \%)$ were prepared by mixing different volumes of deionized water and crude oil samples. Stabilities of the formed emulsion were evaluated by measurements of viscosity using Brookfield dial reading viscometer. Accurate reproducible viscosity measurements were obtained within $1 \%$ of the range used can be made in less than 30 seconds using such viscometer. Viscosity is measured by determining the torque required to spindle the viscometer at constant speed which is immersed in the emulsion sample. This torque is proportional to the viscous force drag on the immersed spindle, and consequently of the viscosity of the emulsion sample.

\section{Results and Discussion}

\subsection{Effect of inorganic solids, wax to asphaltene ratio, and water cut}

Asphaltenes, resins, waxes, and crude oil separated from four different emulsion samples have been used in this study. Molecular mass, density and elemental analysis of these fractions are reported in Table 1.

\subsection{Effect of inorganic solid particles}

Figure 1 through 3 shows the effect of increasing concentration of solid particles (w/w) of $\mathrm{Fe}_{3} \mathrm{O}_{4}$ in various crude oils (RA4, BG75, and Bg366T) on the stability of water-in-oil emulsions. The least stable emulsion is one formed from oil BG75 (Figure 2), however, the most stable emulsion is the one formed from oil BG 366T, Figure 3. It is important to note that sample BG75 has the highest R/A ratio and sample BG 366 has the lowest R/A ratio, Table 2. The general trend observed from these figures is that higher solid content leads to higher degree of stability of the emulsion formed. In other wards, increasing the solid content of $\mathrm{F}_{3} \mathrm{eO}_{4}$ resulted in less amount of water resolved from emulsion upon centrifugation and heating. Small inorganic particles enhance water-in-oil emulsion stability (Sarbar and Wingroove; 1997; Kim et al., 1990; Mc Lean and Kilpatrick, 1997a,b; Gelot, et al., 1984; Yan et al., 1999, 2001; Tambe and Sharma,1993, 1994; Aveyard, et al., 2003; Sztukowski and Yarranton , 2005). The presence of solid particles can add to the stability if the particle sizes are small enough to become interfacially active, with the adsorption of resins and asphaltene from crude oils. The presence of the solid particles adds bulk to the water-oil interfaces and increases the stability of the asphaltene-resin film. Maximum emulsion stabilization effect appears when particles are partially wet by both phases. Hydrophilic particles have contact angles less than $90^{\circ}$ and stabilize oil-in-water emulsion. However, hydrophobic particles have contact angle higher than $90^{\circ}$ and stabilize water-in-oil emulsions.

\subsection{Effect of changing wax to asphaltene ratio}

The role of wax in stabilizing water-in-oil emulsion has been studied via changing the wax to asphaltene (W/A) ratios and determining the amount of water resolved from emulsion in each case using oil RA4 and BG74. Figure 4 and 5 shows the effect of adding various amount of wax extracted from Kuwaiti crude oils on the stability of water-in-crude-oil emulsion. The results obtained from these figures suggest 
that in case of crude oils having high wax content, the formation of wax particles plays an important role when it comes to stabilization of water-in-crude-oil emulsion. Figure 4 and 5 shows that at low wax content (W/A ratio of $0.5: 1$ ) the emulsions were very stable and no water resolved. However, increasing the wax to asphaltene ratio from 1:1 to 2:1 was found to reduce the stability of water-crude oil emulsion.

It is well known that wax particles which are oil wet can precipitate during oil production and transport if the oil temperature fall below the wax crystallization temperature. As long as the wax particles remain oil wet, they remain in the oil phase. However, if asphaltene aggregates adsorb on their surfaces this wettability changes from totally oil wet to mixed wettability. This occurs when the hydrophobic side of asphaltene aggregates adsorb on a wax particle, producing a partially solvated particles. This solvated wax particles would then migrate to the oil water interface contributing to the stability of the emulsion. In this study, this occurs at low W/A ratio below 1:1, where addition of wax promoted the stability of emulsions. Types of wax crystals and their crystalline nature as well as quantity of wax will play a key role on the way and how wax could interfere with oil-water separation process. Normal paraffin wax molecules form thin orthorhombic plates, along with some long needles during their crystallization. Branched, iso-paraffin and cyclo-paraffin waxes also form this plate-like crystal, however, their size remains much smaller. This is due to steric hindrance effect of the side branches and /or cyclic groups, which restrict crystal growth forming microcrystalline structure wax. These small wax crystals posses a large surface area which can contribute to emulsion stability at greater extent than larger crystals resulting from normal paraffins. Ali and Alqam (2000) studied the role, of wax on stability of emulsions formed in Saudi oil fields. They found that wax separated from wells suffering of emulsion problem is predominantly of the high melt microcrystalline type.

The stability of emulsions shows high degree of temperature dependence for crude oils with high wax content. At temperature well above wax crystallization temperature, the oil and aqueous phase separate immediately. While at temperature below the wax crystallization temperature very stable emulsion is formed. It is well known that the solid particles may stabilize the emulsion by hindering coalescence (Mouralle et al., 1998). When the wax crystallization is hindered or changed, the stabilization due to wax particles become less important. The presence of wax particles is not sufficient to stabilize emulsion without the presence of asphaltenes and resins. Asphaltenes and resins are polar molecules have a strong affinity and adsorb on other organic such as wax and inorganic solids, producing emulsion-stabilized films at oil/water interfaces and contributing to the formation of stable emulsions ( Khadim and Sarbar, 1999; Gu et al., 2002).

\subsubsection{Effect of water cut}

In this study, the effect of water cut on the stability of water-in-crude-oil emulsions has reported. The interrelation between viscosity and the stability of emulsion was investigated in this section. The results of the study are presented in term of viscosity of emulsion as a function of water cut at different temperatures. The viscosity measurements were carried out with water cuts in the range of 0 to $60 \%$ at three temperatures, 25,50 and $75^{\circ} \mathrm{C}$.

It is well known that the higher the stability of the emulsion the higher will be its viscosity. Figures 6 through 8 show the effect of water cut on viscosity and hence stability of different crude oil emulsions (BG75, RA4, and BG366) at different temperatures. The rate of increase of the viscosity as a function of water cut is much more pronounced as the temperature is reduced for all the crude oil emulsions. For example, viscosity of emulsion sample prepared from Kuwaiti crude oil RA4 is reduced from 18,000 to $4000 \mathrm{cPs}$ when the temperature was elevated from 25 to $75^{\circ} \mathrm{C}$. It is noticed that the viscosity of water-in-crude-oil emulsion increases with the percentage of water cut until it reached the inversion point where a sharp decline in viscosity takes place. This inversion point for all crude oil considered in this study was found to be at approximately $50 \%$ water cut. This result very well agrees with the range of 28 to $50 \%$ reported by Benayoune et al., 1998. El Gamal et al (2004) also studied viscosity behavior of water-inoil emulsions having water cuts from 10 to $50 \mathrm{wt} \%$. Their results showed that kinematic viscosity steadily increased as a function of increasing the water cut. Increasing the water cut above the inversion point makes the emulsion less stable. Kokal (2005) investigated the effect of water cut on emulsion stability by conducting experiments with emulsion samples in which water cut was varied from $5 \%$ to $80 \%$. This study reported that the emulsions become slightly less tight as the water cut increased. 


\section{Summary and conclusion}

Asphaltenes, resins, waxes and crude oils separated from various emulsion samples collected from Burgan oil field in Kuwait have been used to study the effect of inorganic solid $\left(\mathrm{F}_{3} \mathrm{eO}_{4}\right)$ on the stability of water-in-crude oil emulsions. The results indicate that in the presence of asphaltene and resin in crude oil increasing the solid content leads to tighter emulsions. The presence of fine solids adds bulk to the wateroil interface and increases the stability of asphaltene-resin film.

The role of wax to asphaltene ratio in stabilizing water-in-oil emulsions has also been studied. The experimental results showed that at low wax to asphaltene (W/A) content the emulsions were very stable and no significant amount of water resolved from emulsions. At high W/A ratio above 1:1, the stability of water-crude oil emulsion is reduced. The stability of emulsions having high wax content is highly temperature dependent. At temperature well above wax crystallization temperature, the oil and aqueous phase separate immediately. While, at temperatures below the wax crystallization temperature, very stable emulsion is formed. The presence of wax particles alone is not sufficient to stabilize water-in-oil emulsions. Asphaltenes and resins are polar molecules having a strong affinity and adsorb on other solid particles such as wax producing stable water-in-oil emulsions.

The effect of water cut on the stability of water-in-crude oil emulsions has also been investigated at various temperatures $\left(25\right.$ to $\left.75^{\circ} \mathrm{C}\right)$. Stability and hence viscosity of emulsion increases as a function of increasing the water cut, especially at low temperature. The viscosity of water-in-oil emulsion increases with the percentage of water in the emulsion until it reaches the inversion point where a sharp decline in viscosity takes place. The inversion point for the crude oils considered in this study was found to be approximately at $50 \%$ water cut. Increasing water cut above the inversion point makes the emulsion less stable.

\section{Acknowledgements}

This project has been financially supported by Kuwait Foundation for Advancement of Sciences, Grant No. KFAS 2000-09-02, "Role of asphaltene, reins, and wax in the stability of water/oil emulsions in Kuwaiti Crudes". Special thanks go to the Research Administration at Kuwait University for administrating the project, providing assistance in manpower and equipments. The authors also thanks Kuwait Oil Company (KOC) for providing emulsion samples used in this research project. Elsharkawy thanks the Department of Chemical and Petroleum Engineering, University of Calgary for providing computing and other research facilities during his sabbatical leave.

\section{References}

Ali, M.F., Alqam, M.H., 2000. The role of asphaltenes, resins and other solids in the stabilization of water in oil emulsions and its effect on oil production in Saudi oil fields, Fuel, 79, 1039-1316.

Aske, N., Kallevik, H., Sjöblom, J. Water-in-crude oil emulsion stability by electrical field measurements. Correlation to physico-chemical parameters and near-infrared spectroscopy. J. Petrol. Sci Eng. 36, 117.

Aveyard, R., Binks, B.P., and John H. Clin, J.H., 2003.Emulsions stabilized solely by colloidal particles. Adv. Colloid Interface Sci. 100-102, 503-546.

Benayoune, M., Khezzar, L., Al-Rumhy, M., 1998. Viscosity of water in oil emulsions. J. Petrol Sci, Tech., $16,767-784$.

El Gamal, M., Mohamed, A. O., and Zekri, A.Y., 2005. Effect of asphaltene, carbonate, and clay mineral contents on water cut determination in water-oil emulsions, J. Pet. Sci. and Eng., 46,209-224.

Elsharkawy, A.M., Al-Sahaf, T. A., Fahim, M.A., 2000. Wax deposition from Middle East crudes, Fuel, 79, 1074-1055.

Elsharkawy, A.M., Al-Sahaf, T. A., Fahim, M.A., 2005. Characterization of Asphaltenes and Resins Separated from Water-in-Crude Oil Emulsions formed in Kuwaiti Oil Fields. Submitted to J. Petrol Sci. Technol.

Fingas, M., Fieldhouse, B. 2004. Formation of water-in-oil emulsions and application to oil spill modeling. J. Hazardous materials, 107, 37-50.

Gafonova, O.V. and Yarranton, H.W., 2001. The stabilization of water-in-hydrocarbon emulsions by asphaltenes and resins. J. colloidal interface Sci. 241,469-478.

Gelot, A., W. Friesen, W., Hamza, H. A., 1984. Emulsification of oil and water in the presence of finely divided solids and surface-active agents. Colloids Surf. 12, 271-303. 
Gu, G., Xu, Z., Nandakumar, K., Masliyah, J. K, 2002. Influence of water-soluble and water-insoluble natural surface active components on the stability of water-in-toluene-diluted bitumen emulsion, Fuel, 81, 1859-1869.

Khadim, M.A., Sarbar, M.A. 1999. Role of asphaltene and resins in oil field emulsions J. Petroleum Sci. Eng., 23,213-222.

Khristov, Khr., Taylor, S.D., Masliyah, J.H., 2000. Thin liquid film technique: application to water-in-oil bitumen emulsion films. Colloid Surf., A Physicochem. Eng. Asp. 174,183-196.

Kim, S.T., Boudh-Hir, M.E., Mansoori, G.A., 1990. The role of asphaltene in the wettability reversal. Proceedings of the 1990 Annual Convention of the Society of Petroleum Engineers SPE paper No. 20700.

Kokal, S. and Wingrove, M. 2000. Emulsion separation index: from laboratory to field cases. Paper SPE 63165. Proceedings of the SPE Annual Technical Conference and Exhibition, Dallas, TX, USA, October, 2000.

Kokal, S. and Al-Ghamdi, A., 2005. Oil-water separation experience from a large oil field. Paper SPE 9338

Proceedings of the $14^{\text {th }}$ SPE Middle East Oil\& Gas Show and Conference held in Bahrain, March 12-15, 2005.

Li, M., Lin, M., Wu, Z., Christy, A.A. 2004. The influence of $\mathrm{NaOH}$ on the stability of paraffinic crude oil emulsion. Fuel, 84, 183-187.

Li, M., Xu, M., Ma, Y., Wu, Z., Christy, A.A. 2002. The effect of molecular parameters on the stability of water-in-oil emulsions studied by IR and UV spectroscopy. 2002. Colloids Surf., A Physicochem. Eng. Asp. 197, 193-201.

Mc Lean, J.D., Kilpatrick, P.K., 1997a. Effects of asphaltene solvency on the stability of water-in-oil emulsion, J. Colloid Interface Sci.189, 242-253.

Mc Lean, J.D., Kilpatrick, P.K., 1997b. Effects of asphaltene aggregation in the model heptane-toluene mixtures on the stability of water-in-oil emulsion. J. Colloid Interface Sci.196, 23-34.

Midttun, Ö, Kallevik, H., Sjöblom, J, Kvalheim, O. 2000. Multivariate screening analysis of water-in-oil emulsions in high external electric field as studied by means of dielectric time domain spectroscopy. J. Colloid Interface Sci, 227, 262-271.

Mouralle, O., Sjoblom, J., Peytavy, J.L., 1998. Stability of water-in- crude oil emulsions: role played by the state of salvation of asphaltene and waxes. J. Dispersion Sci. Tech. 19, 339-367.

Ramos, A. C. S., Haraguchi, L., Notrispe, F.R., Loh, W. Mohamed, R.S., 2001. Interfacial and colloidal behavior of asphaltene obtained from Brazilian crude oils. J. Pet. Sci. Eng., 32, 201-216.

Salou, M., Siffert, B., Jada, A., 1998. Study of the stability of bitumen emulsions by application of DLVO theory. Colloids Surf., A Physicochem. Eng. Asp. 142, 9-16.

Sarbar, M., Wingroove, M.D. et al. 1997. Physical and chemical characterization of Saudi Arabian crude oil emulsions. Paper SPE 38817. Proceedings of the SPE Annual Technical Conference and Exhibition, San Antonio, TX, USA, October 5-8.

Schorling, P.-C., Kessel, D.G., Rahimian, I., 1999. Influence of the crude oil resin/ asphaltene ratio on the stability of oil/water emulsions. Surfaces A: Physicochemical Eng. Aspects, 152, 95-102.

Spiecker, P.M., Gawrys, K.L., Trail, C. B., Kilpatrick, P.K. 2003. Effects of petroleum resins on asphaltene aggregation and water-in-oil emulsion formation. Colloids Surf., 220, 9-27.

Sullivan, A.P., Kilpatrick, P.K., 2002. The effect of inorganic solid particles on water and crude oil emulsion stability. Ind. Eng. Chem. Res. 41, 2289-3404.

Sztukowski, D.M., Yarranton, H.W., 2005. Oilfield solids and water in oil emulsion stability. J. Colloid Interface Sci. 285, 821-833

Tambe, D. , Sharma, M.M. 1994. Factors Controlling the Stability of Colloid-Stabilized Emulsions: II. A Model for the Rheological Properties of Colloid-Laden Interfaces. J. Colloid Interface Sci. 162, 1-10.

Tambe, D. E., Sharma, M.M., 1993. Factors Controlling the Stability of Colloid-Stabilized Emulsions: I. An Experimental Investigation. J. Colloid Interface Sci. 157, 244-25.

Xia, L., Lu, S., Cao, G., 2004. Stability and demulsification of emulsions stabilized by asphaltenes and resins. J. Colloid Interface Sci. 271, 504-506.

Yaghi, B. 2003. Rheology of oil-in-water emulsions containing fine particles. J. petroleum Sci, Eng., 40, 103-110.

Yan, N., Gray, M. R., Masliyah, J. H., 2001. On water-in-oil emulsions stabilized by fine solids. Colloids Surfaces A: Physicochemical Eng. Aspects, 193, 97-107. 
Yan, Z., Elliott, J.A. W., Masliyah, J.H., 1999. Roles of Various bitumen components in the stability of water-in-diluted-bitumen emulsion. J. Colloid Interface Sci., 220,329-337.

Yarranton, H.W., Hussein, H., Masliyah, J.H, 2000. Water-in-hydrocarbon emulsions stabilized by asphaltenes at low concentrations. J. Colloid Interface Sci. 228, 52-63.

Table 1-Properties of oils, asphaltenes, and resins used to prepare emulsion samples

\begin{tabular}{|c|c|c|c|c|c|c|c|c|c|}
\hline \multirow[t]{2}{*}{ sample } & \multirow{2}{*}{$\begin{array}{c}\text { Molar } \\
\text { mass }\end{array}$} & \multirow{2}{*}{$\begin{array}{l}\text { Density } \\
@ 20^{\circ} \mathrm{C}\end{array}$} & \multirow{2}{*}{$\begin{array}{l}\mathrm{H} / \mathrm{S} \\
\text { ratio }\end{array}$} & \multirow{2}{*}{$\begin{array}{l}\mathrm{H} / \mathrm{C} \\
\text { ratio }\end{array}$} & \multicolumn{5}{|c|}{ Elemental analysis } \\
\hline & & & & & $\mathrm{C} \%$ & $\mathrm{H} \%$ & $\mathrm{~N} \%$ & $\mathrm{~S} \%$ & $\mathrm{O} \%$ \\
\hline \multicolumn{10}{|l|}{ Oils } \\
\hline BG47T & 399 & 0.9294 & 4.1497 & 1.6556 & 82.645 & 11.483 & 0.0669 & 2.7672 & 3.0379 \\
\hline BG185T & 392 & 0.9255 & 4.4565 & 1.7038 & 83.026 & 11.872 & 0.1043 & 2.6640 & 2.3337 \\
\hline BG321T & 395 & 0.9203 & 3.9984 & 1.6231 & 80.594 & 10.978 & 0.1006 & 2.7456 & 5.5818 \\
\hline BG366T & 403 & 0.9383 & 4.1173 & 1.6243 & 81.714 & 11.139 & 0.0705 & 2.7054 & 4.3711 \\
\hline \multicolumn{10}{|l|}{ Asphaltenes } \\
\hline A-BG47T & 4246 & 1.2374 & 1.1630 & 1.1623 & 76.50 & 7.463 & 1.159 & 6.41 & 8.454 \\
\hline A-BG185T & 4052 & 1.2415 & 1.1498 & 1.1487 & 76.66 & 7.391 & 1.221 & 6.42 & 8.295 \\
\hline A-BG321T & 4432 & 1.2256 & 1.1197 & 1.1706 & 78.67 & 7.729 & 1.233 & 6.91 & 5.460 \\
\hline A-BG366T & 3997 & 1.2430 & 1.1324 & 1.1541 & 76.85 & 7.443 & 1.255 & 6.57 & 7.883 \\
\hline \multicolumn{10}{|l|}{ Resins } \\
\hline R-BG47T & 1150 & 1.1047 & 1.7208 & 1.3684 & 79.22 & 9.098 & 1.143 & 5.29 & 5.251 \\
\hline R-BG185T & 984 & 1.0859 & 1.6387 & 1.3629 & 79.36 & 9.077 & 1.167 & 5.54 & 4.856 \\
\hline R-BG321T & 1121 & 1.1099 & 1.5628 & 1.3561 & 90.98 & 9.216 & 1.156 & 5.89 & 2.753 \\
\hline R-BG366T & 992 & 1.0860 & 1.5407 & 1.3595 & 81.09 & 9.252 & 1.181 & 6.05 & 2.468 \\
\hline
\end{tabular}

Table 2-Resin to asphaltene (R/A) ratio for various emulsion samples

\begin{tabular}{c|c}
\hline Sample & Resins to asphaltene (R/A) ratio \\
\hline BG 366 & 4.4 \\
BG 75 & 12.4 \\
RA4 & 9.0 \\
\hline
\end{tabular}


Fig 1

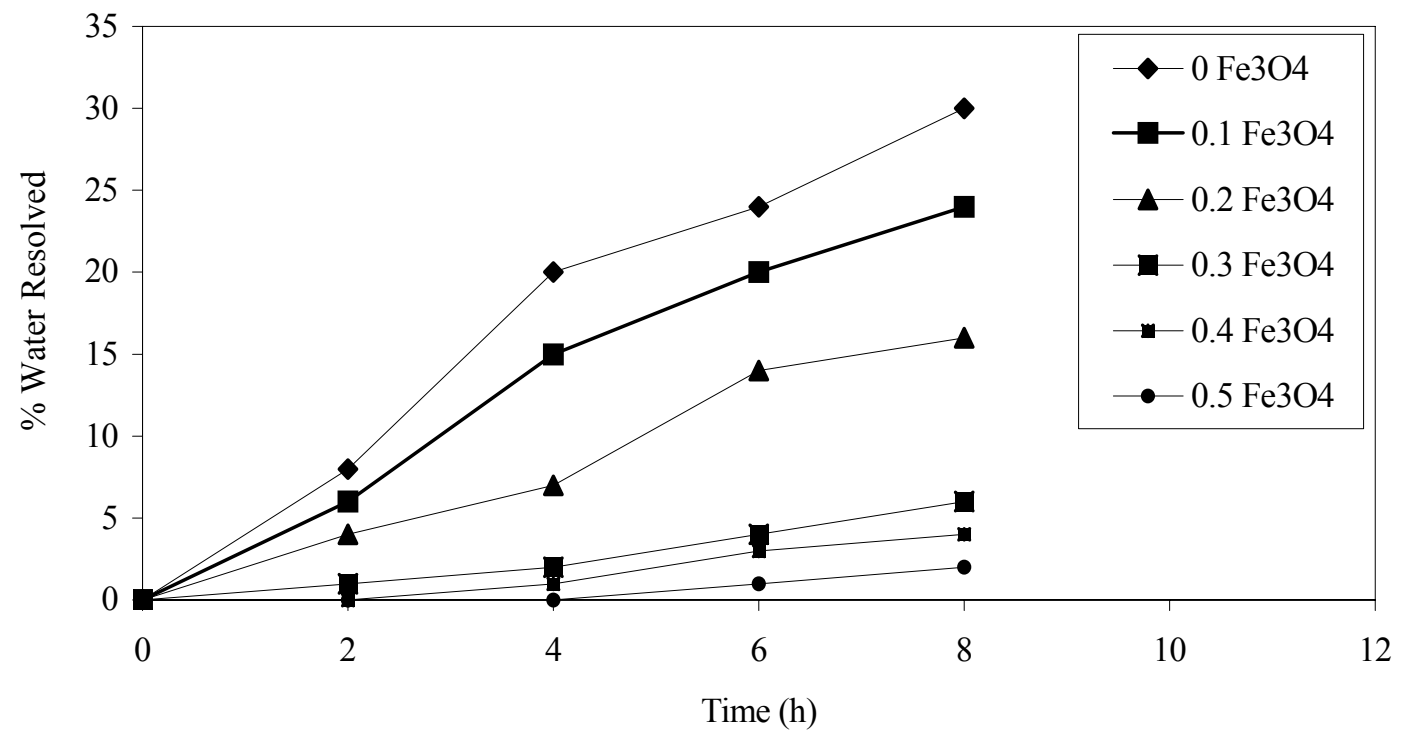

Figure 1. Effect of Inorganic Solids on Stability of Water-in-Crude Oil Emulsion (RA 4).

Fig 2

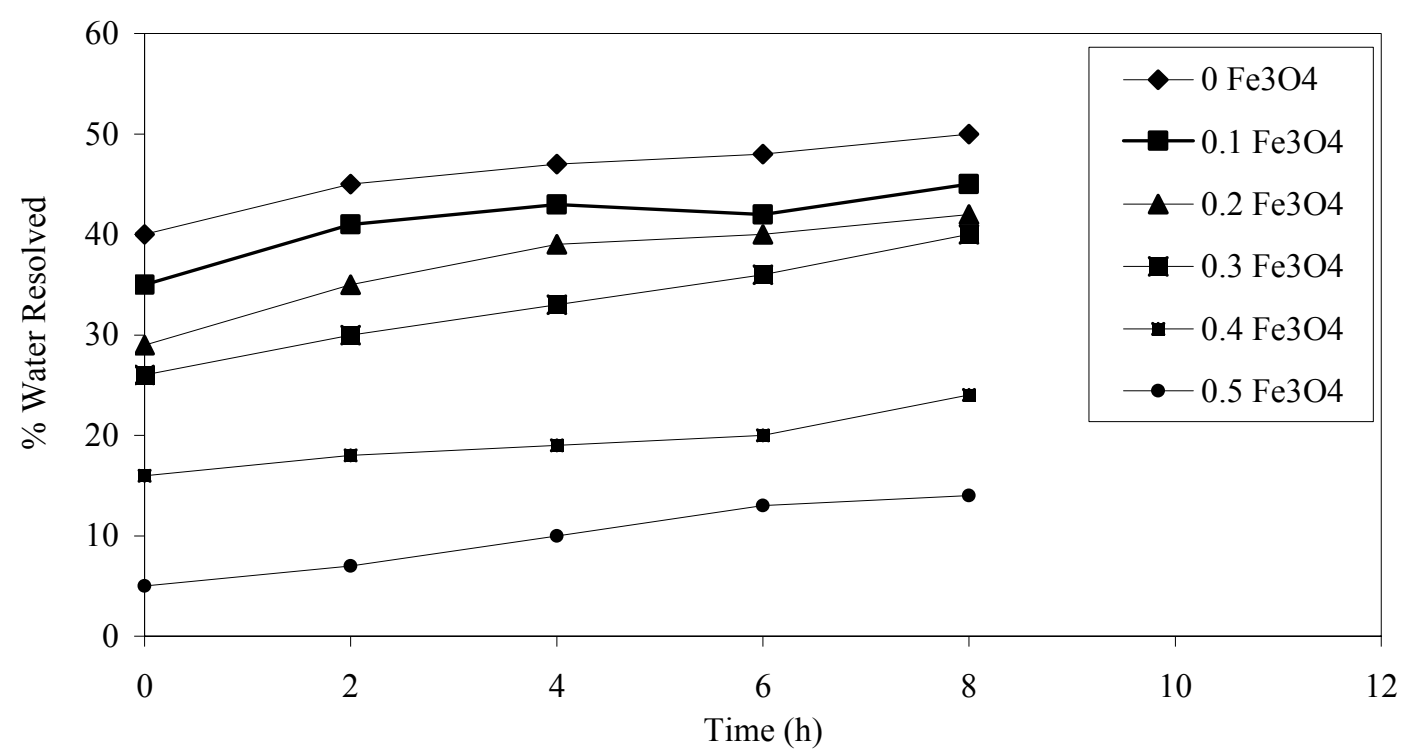

Figure 2. Effect of Inorganic Solids on Stability of Water-in-Crude Oil Emulsion (BG 75). 
Fig 3



Figure 3. Effect of Inorganic Solids on Stability of Water-in-Crude Oil Emulsion (BG 366 T).

Fig 4

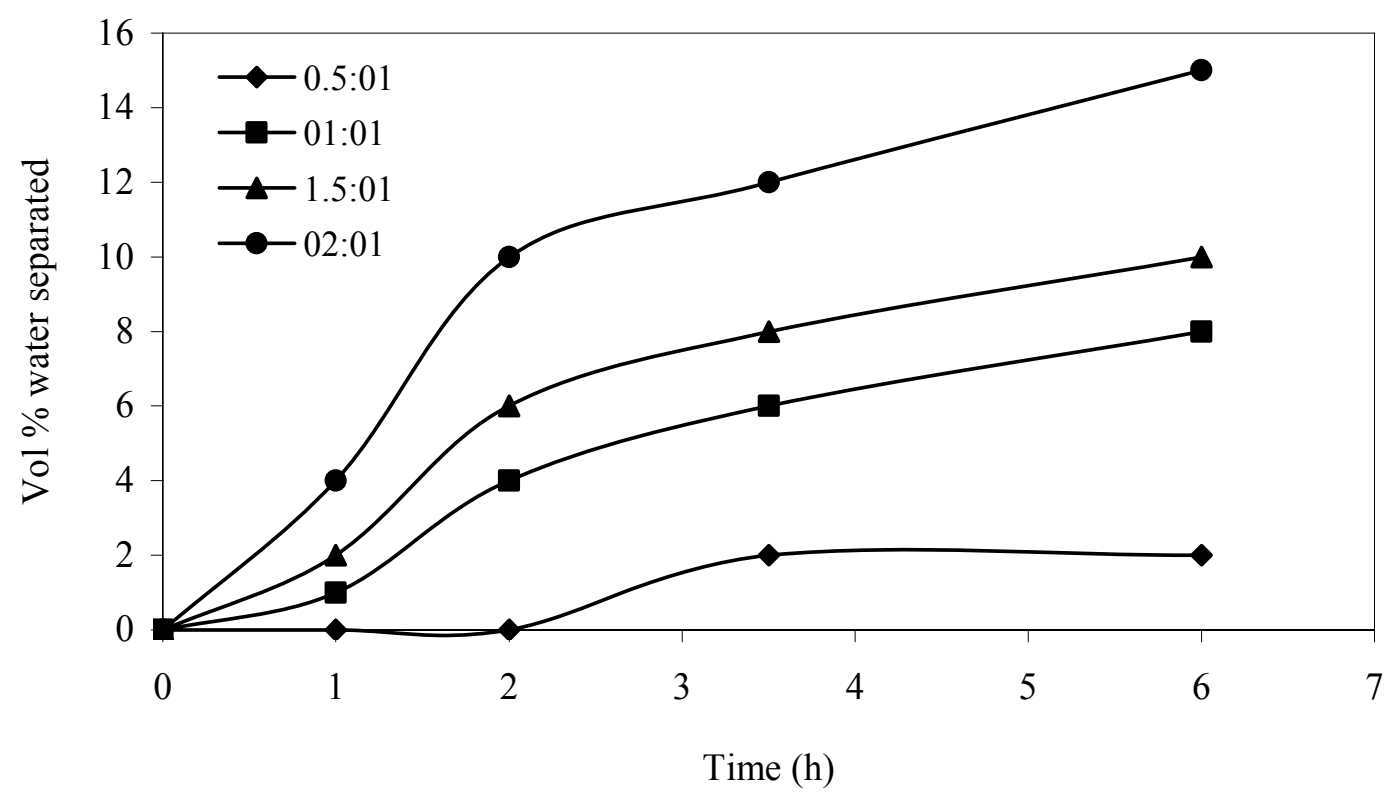

Figure 4. Effect of Wax to asphaltene ratio on water-in-oil emulsion (RA 4). 
Fig5

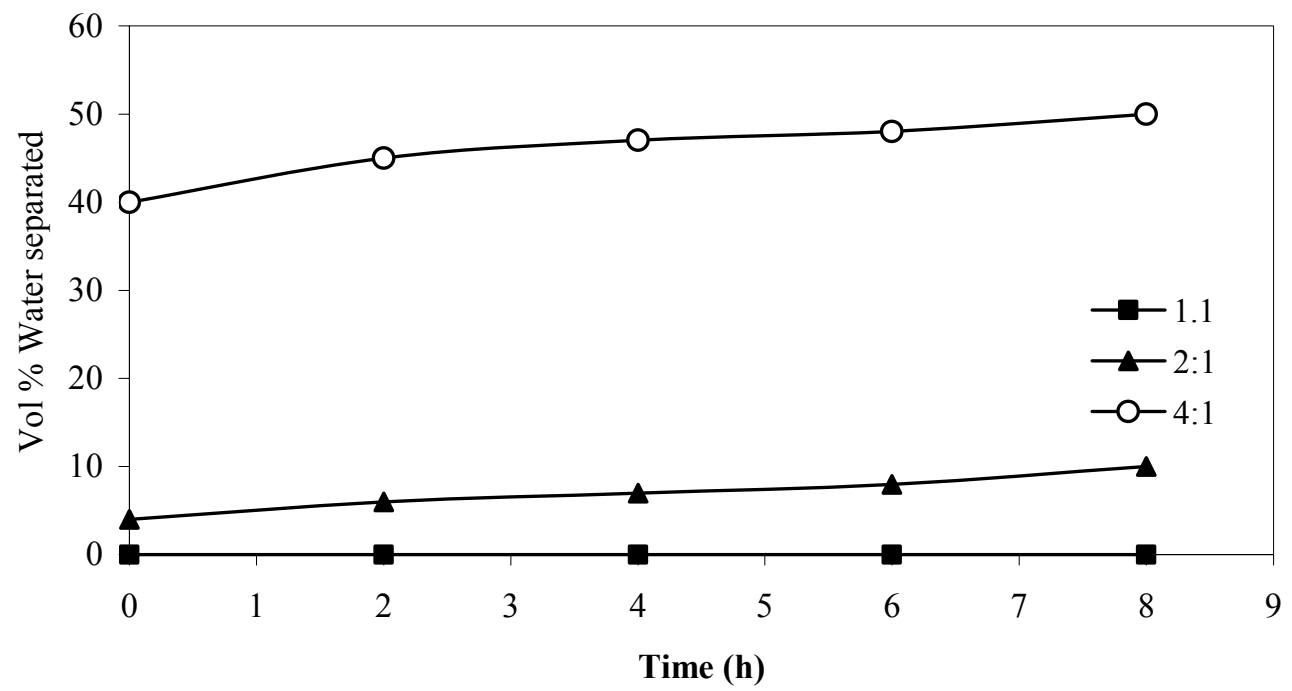

Figure 5. Effect of Wax to Asphaltene ratio on stability of water -in-crude oil emulsion (BG 75)

Fig 6

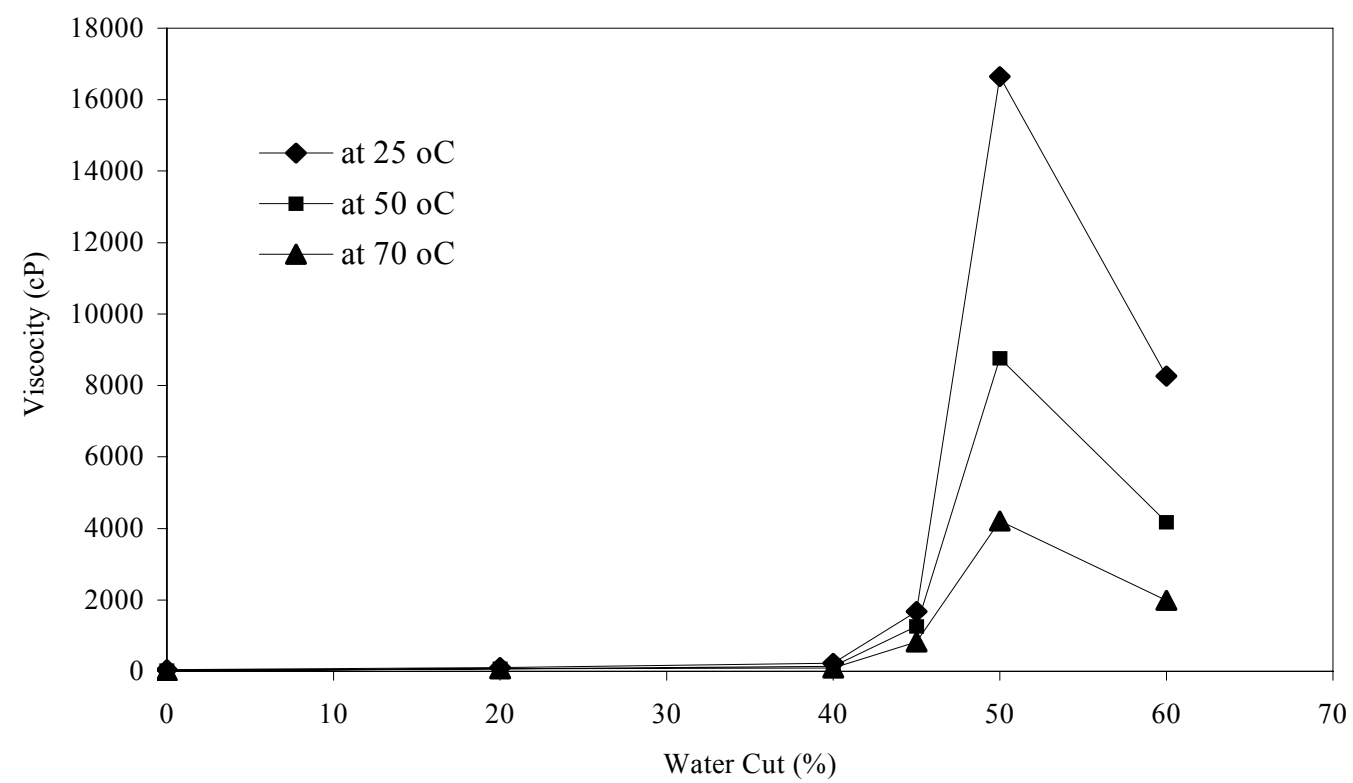

Figure 6. Effect of Water Cut on Viscocity of Crude Oil (RA 4) at 25, 50 and 70 oC. 
Fig 7

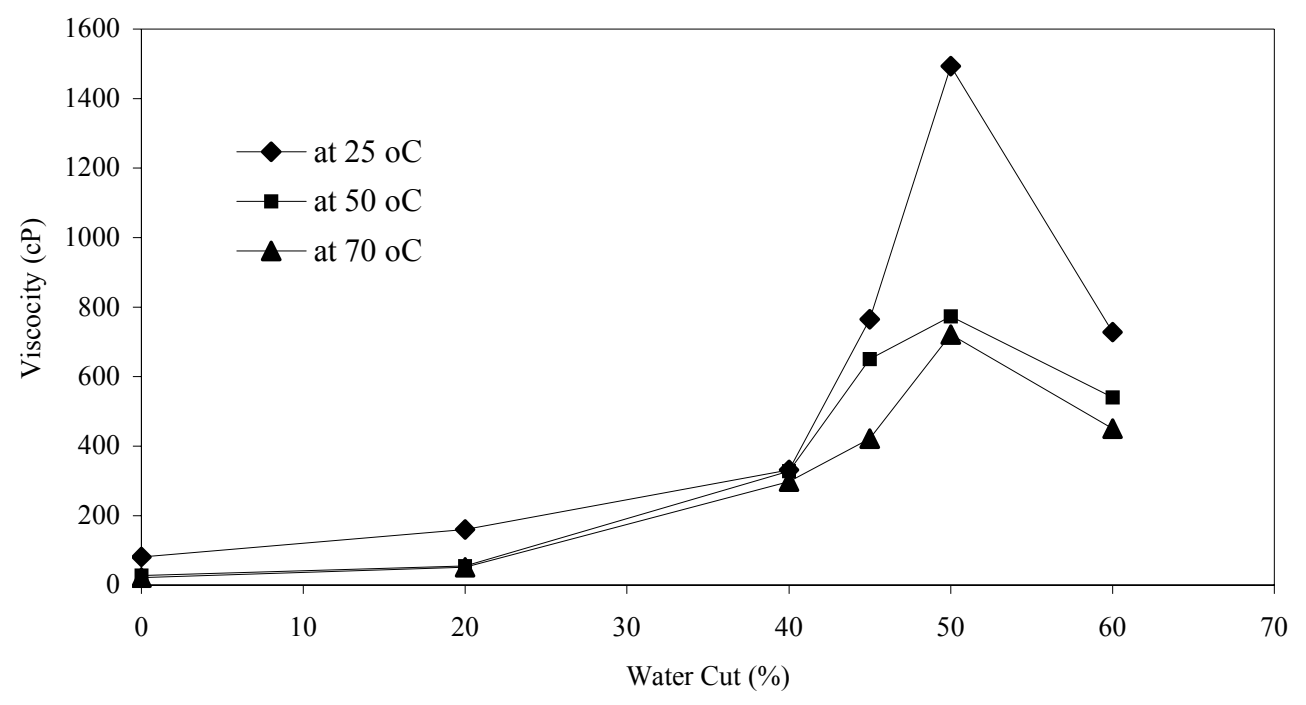

Figure 7. Effect of Water Cut on Viscocity of Crude Oil (BG 75) at 25, 50 and $70 \mathrm{oC}$.

Fig 8

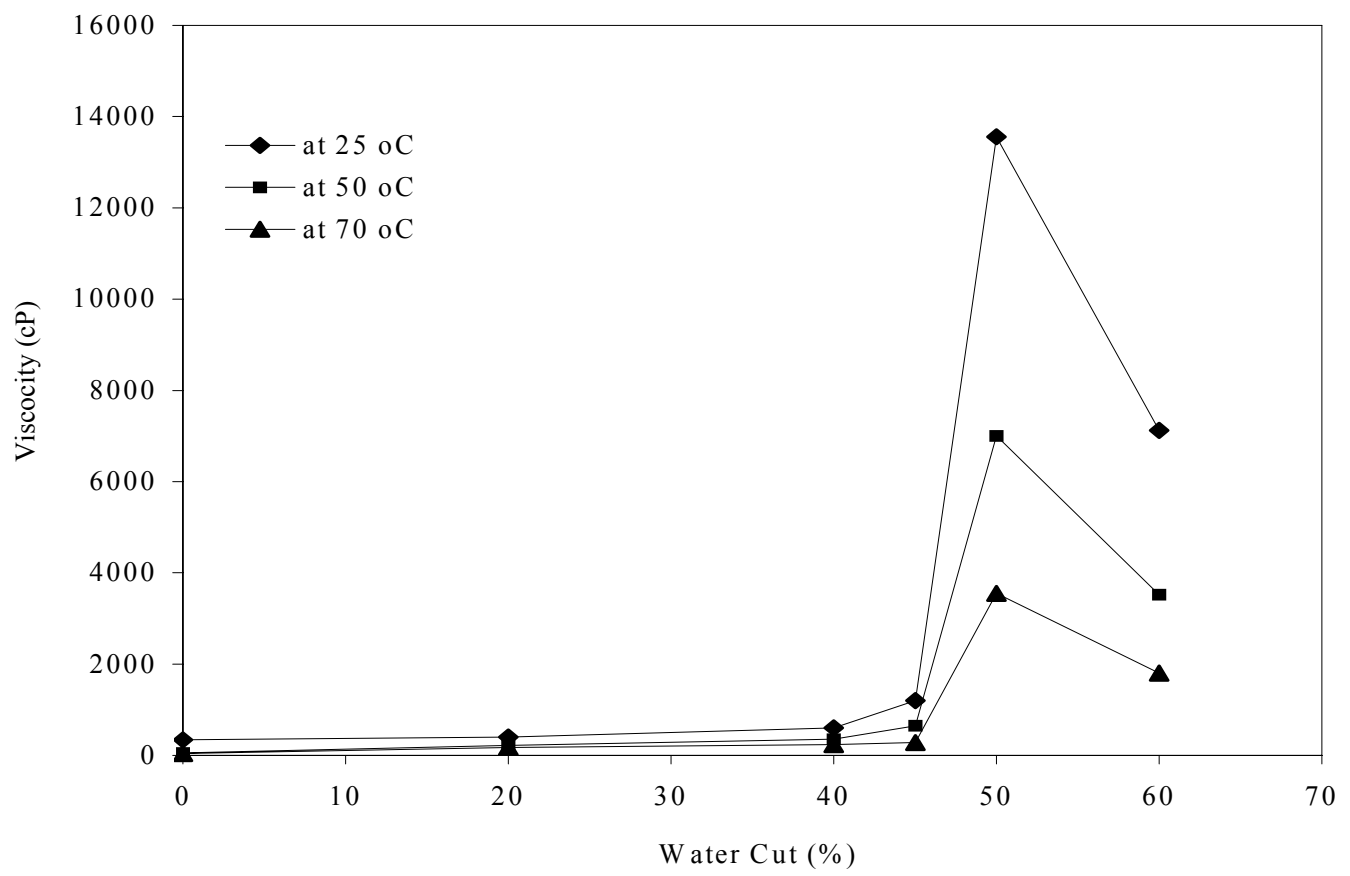

Figure 8. Effect of W ater Cut on Viscocity of Crude Oil BG 366 at 25, 50 and 70 oC. 